Fanta Reine Sheirita TIÉtıambou ${ }^{1}$ Anne Mette LYKKE ${ }^{2}$

Gabin KorbÉOGO3

Adjima ThIOMBIANO

Amadé OUÉdRAOGO ${ }^{1}$

${ }^{1}$ Université Ouaga I Joseph Ki-Zerbo UFR en Sciences de la Vie et de la Terre

Laboratoire de biologie et écologie végétales

03 BP 7021, Ouagadougou 03

Burkina Faso

${ }^{2}$ Aarhus University

Departement of Bioscience

Silkeborg

Danemark

3 Université Ouaga I Joseph Ki-Zerbo Département de sociologie 01 BP 1392, Ouagadougou 01 Burkina Faso

\section{Perceptions et savoirs locaux sur les espèces oléagineuses locales dans le Kénédougou, Burkina Faso}

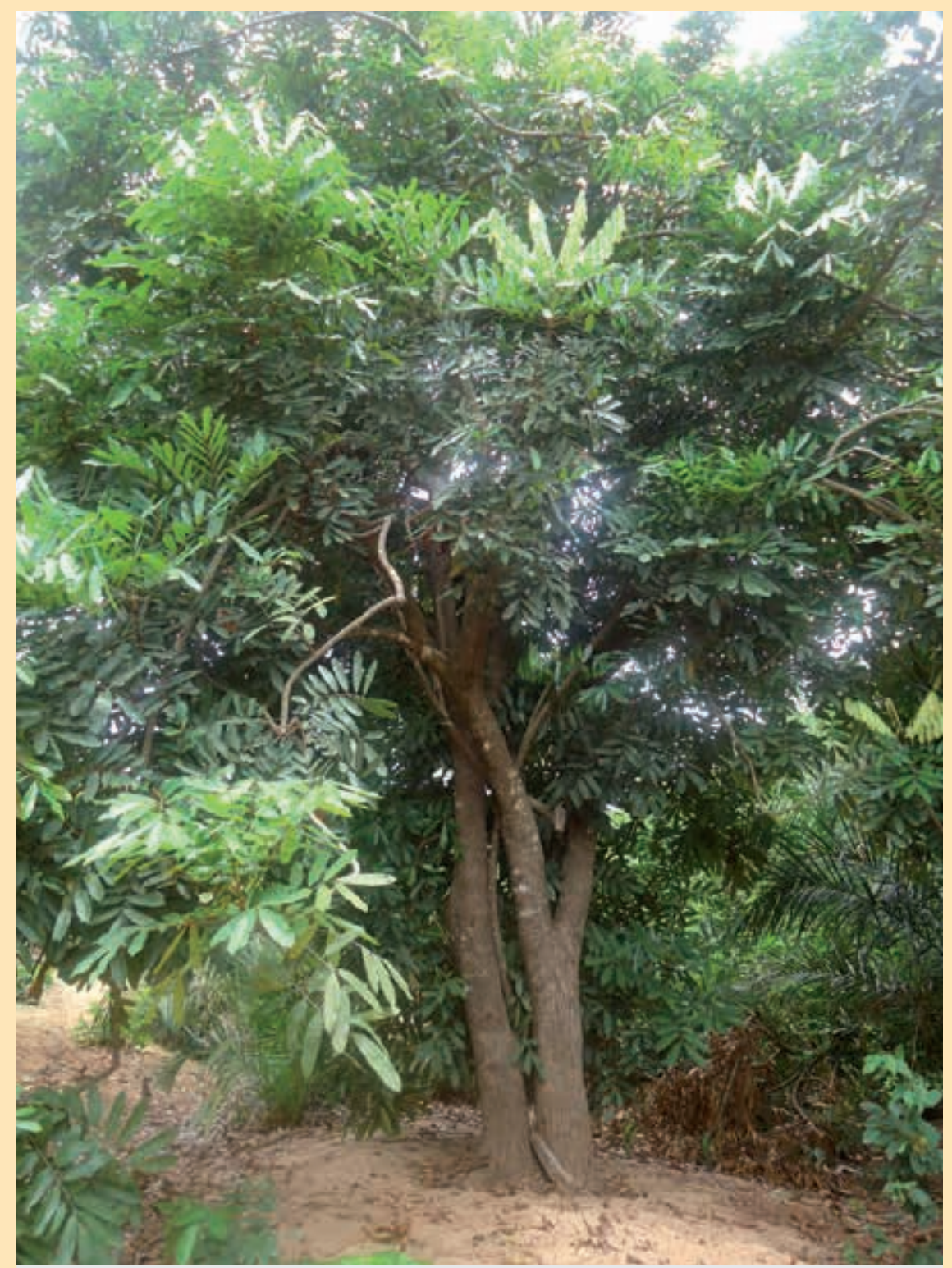

Photo 1.

Pied de Carapa procera dans son milieu naturel de galerie forestière.

Photo B. Lankoandé. 


\section{RÉSUMÉ}

\section{PERCEPTIONS ET SAVOIRS LOCAUX SUR LES ESPÈCES OLÉAGINEUSES LOCALES DANS LE KÉNÉDOUGOU, BURKINA FASO}

Les plantes oléagineuses locales offrent des usages multiples et constituent une source de revenus pour les communautés rurales. L'objectif de notre étude est de comprendre les perceptions et les savoirs des populations de la province du Kénédougou (Burkina Faso) concernant ces plantes et leurs huiles. La méthodologie utilisée est une combinaison de l'approche participative sociale et d'enquêtes ethnobotaniques. Au total, 336 personnes ont été interrogées au sein de quatre groupes communautaires (Toussian, Siamou, Bolon et Peulh) à travers 14 villages. Les résultats révèlent 11 plantes oléagineuses connues, parmi lesquelles quatre représentent $94 \%$ de l'ensemble des citations. Le savoir sur les espèces ne varie significativement que selon l'âge $\left(X^{2}=8,11 ; p=0,0173\right)$. Ce sont les adultes et les aînés qui disposent plus de savoir sur ces espèces. Les femmes sont les principales détentrices des savoirs sur les techniques d'extraction des huiles et leur transformation $\left(X^{2}=19,79 ; p<0,001\right)$. Ces techniques sont connues pour sept espèces mais elles ne sont effectivement appliquées que pour l'extraction des huiles de quatre espèces : Vitellaria paradoxa, Elaeis guineensis, Carapa procera et Lophira lanceolata. Les huiles des 11 espèces citées sont principalement utilisées dans la médecine (47\%), l'alimentation (37\%) et la cosmétique (16\%). La préférence des communautés pour les huiles est influencée par leurs utilisations mais également par le savoir sur la technique d'extraction, la facilité de cette technique et le rendement en huile extractible de la graine. Les valeurs du Facteur consensus d'informateurs (68\%) révèlent que les espèces que les populations consentent à préserver sont celles qui sont les plus utilitaires. La promotion de l'huile de ces plantes pourrait être un atout pour leur préservation.

Mots-clés : savoirs locaux, plantes locales, plantes oléagineuses, préférences communautaires, valeur d'utilisation, Burkina Faso.

\section{ABSTRACT}

\section{RESUMEN}

\section{LOCAL PERCEPTIONS AND KNOWLEDGE OF LOCAL OIL-SEED PLANTS IN BURKINA FASO’S KÉNÉDOUGOU PROVINCE}

Local oil-producing plants have many different uses and are a source of income for rural communities. The aim of our study is to understand local perceptions and knowledge of these species and the oil they produce in Burkina Faso's Kénédougou Province. The methodology used combines a participatory social approach with ethnobotanical surveys. We interviewed a total of 336 people from 4 community groups (Toussian, Siamou, Bolon and Fulani) across 14 villages. The results show that 11 oil-seed plants are well known, four of which account for $94 \%$ of all citations. Significant variations in knowledge on the rarely cited species are age-related $\left(X^{2}=8.11 ; p=0.0173\right)$. Adults and elderly people are more knowledgeable.Women have the most knowledge of oil extraction and processing techniques $\left(\mathrm{X}^{2}=19.79\right.$; $p<0.001)$. These techniques are known for seven species, but are only applied to extract oil from four of them, Vitellaria paradoxa, Elaeis guineensis, Carapa procera and Lophira lanceolata. The oils from all the 11 cited species are mainly used to prepare medicines ( $47 \%$ ), food (37\%) and cosmetics (16\%). Preferences for different oils among the communities are influenced by their uses but also by knowledge of extraction techniques, their ease of use and the oil yield that can be extracted from the seeds. The figure for the informant consensus factor (68\%) shows that the species that communities are willing to preserve are those that are most useful to them. Promoting the oils produced from these plants could be a key to their preservation.

Keywords: local knowledge, local plants, oil-seed plants, community preferences, use value, Burkina Faso.

\section{PERCEPCIONES Y SABERES LOCALES DE LAS ESPECIES OLEAGINOSAS LOCALES EN KÉNÉDOUGOU, BURKINA FASO}

Las plantas oleaginosas locales brindan usos múltiples y suponen una fuente de ingresos para las comunidades rurales. El objetivo de este estudio consiste en comprender las percepciones y saberes de los pueblos de la provincia de Kénédougou (Burkina Faso) sobre estas plantas y sus aceites. La metodología empleada es una combinación del enfoque participativo social con encuestas etnobotánicas. Se entrevistó a 336 personas pertenecientes a cuatro grupos comunitarios (Toussian, Siamou, Bolon y Fulani) de 14 aldeas. Los resultados revelan que se conocen 11 plantas oleaginosas, cuatro de las cuales representan el $94 \%$ del total de menciones. El conocimiento de las especies sólo varía significativamente con la edad $\left(X^{2}=8,11 ; p=0,0173\right)$. El conocimiento de adultos y ancianos mejora dependiendo de la edad. Las mujeres son las principales depositarias de los saberes relativos a las técnicas de extracción de aceites y su transformación $\left(X^{2}=19,79 ; p\right.$ $<0,001)$. El conocimiento de estas técnicas abarca siete especies, pero en la práctica sólo se aplican para la extracción de aceite de cuatro especies: Vitellaria paradoxa, Elaeis guineensis, Carapa procera y Lophira lanceolata. Los aceites de las 11 especies citado se usan principalmente para medicina (47\%), alimentación (37\%) y cosmética (16\%). La preferencia de las comunidades por los aceites depende de sus usos, pero también del conocimiento y sencillez de la técnica de extracción y del rendimiento de aceite extraído de la semilla. Los valores del Factor de Consenso de Informantes (68\%) revelan que las especies que los pueblos aceptan proteger son las que se consideran más útiles. La promoción del aceite de estas plantas podría suponer una baza para su protección.

Palabras clave: saberes locales, plantas locales, plantas oleaginosas, preferencias comunitarias, valor de uso, Burkina Faso. 


\section{Introduction}

Les plantes locales sont une source de nombreux produits utilisés sur le plan alimentaire, médicinal, cosmétique et artisanal. Ces produits sont exploités par les populations locales comme moyens de subsistance et comme source de revenus (Vodouhê et al., 2009). Au Burkina Faso, ces produits entrent dans l'alimentation et l'équilibre nutritionnel de plus de $43 \%$ des ménages ruraux et leur procurent $23 \%$ des revenus et de l'emploi (APFNL, 2011). Pour ce qui concerne les plantes oléagineuses, les productions issues des matières grasses telles que les huiles et les beurres employés dans l'alimentation, la cosmétique, la médecine et la bioénergie (photo 1) représentent pour le Burkina Faso des opportunités réelles d'amélioration de son produit intérieur brut à travers l'exportation (MEF, 2010). À cet effet, Ouédraogo et al. (2013) ont recensé 28 espèces de plantes oléagineuses locales parmi lesquelles 16 sont souvent utilisées traditionnellement par les populations burkinabè pour divers besoins. Aujourd'hui, les utilisations et les productions issues des espèces oléagineuses font l'objet d'un regain d'intérêt ethnobotanique (Belem et al., 2008 ; Weber et al., 2010 ; Ouédraogo et al., 2013 ; Ouédraogo et al., 2014) et socio-économique (Saussey et al., 2008). Certaines filières oléagineuses sont assez développées et déjà bien connues en Afrique comme le karité (Vitellaria paradoxa C. F. Gaertn.) et le palmier à huile (Elaeis guineensis Jacq.) (Schreckenberg, 2004 ; Saussey et al., 2008 ; Kaboré et al., 2012). D'autres le sont moins car la production et la consommation de leur huile restent marginales. La valorisation et la promotion de ces plantes s'imposent donc (photo 1) ; mais cela ne peut se faire sans la prise en compte des savoirs locaux (Lynam et al., 2004 ; Sheil et Liswanti, 2006 ; Bognounou et al., 2009).

De nombreux travaux ont été réalisés sur les utilisations des plantes locales en général au Burkina Faso (Mbayngone et al., 2008 ; Traoré et al., 2011 ; Zerbo et al., 2011). D’autres se sont intéressés aux préférences de la population en matière d'utilisation des espèces (Sheil et Liswanti, 2006 ; Belem et al., 2008 ; Ouédraogo et al., 2013). Cependant, les savoirs relatifs aux utilisations et aux préférences des huiles des plantes locales restent limités, voire inexistants. Les études sur ces savoirs locaux emploient les méthodes de l'ethnobotanique quantitative et qualitative (Cotton, 1996) pour la détermination des préférences de la population en matière d'utilisation des espèces. Mais ces méthodes ne permettent pas d'évaluer l'importance que pourraient avoir les espèces non exploitées à cause de leur faible abondance ou de leur rareté dans une localité (Mathur et Sundaramoorthy, 2013).

Le but de notre étude est d'appréhender les perceptions et les savoirs locaux des populations sur les plantes oléagineuses locales dans une perspective de développement orienté vers le marché de leurs productions. Elle a été conduite dans la province du Kénédougou, dans l'Ouest du Burkina Faso. Le choix de cette localité se justifie par le fait qu'elle fait partie des zones à haut potentiel de produits forestiers non ligneux (PFNL) (APFNL, 2011) et qu'elle se situe dans une partie du pays où la diversité floristique est l'une des plus élevées (Schmidt et al., 2005). Les objectifs spécifiques de l'étude sont les suivants :

- identifier les facteurs socio-ethniques qui influencent les savoirs des populations sur les plantes oléagineuses locales ;

- déterminer les préférences des communautés rurales sur les huiles des espèces identifiées ;

- évaluer les valeurs d'usage des plantes oléagineuses locales.

\section{Méthodes}

\section{Zone d'étude}

La zone d'étude couvre la province du Kénédougou située dans l'Ouest du Burkina Faso. Cette province se situe entre $10^{\circ} 10^{\prime}$ et $12^{\circ} 05^{\prime}$ de latitude Nord et $4^{\circ} 30^{\prime}$ et $5^{\circ} 30^{\prime}$ de longitude Ouest (figure 1). Elle appartient au secteur phytogéographique sud-soudanien (Fontès et Guinko, 1995) et s'étend sur une superficie de $8403 \mathrm{~km}^{2}$. Le climat est caractérisé par deux saisons contrastées, l'une humide de mai à octobre et l'autre sèche. La pluviométrie annuelle moyenne, de 1991 à 2010, est de 1150 mm. Deux des quatre principaux cours d'eau du Burkina Faso, à savoir le Mouhoun et la Comoé, y prennent leur source. Le relief est dominé par une vaste pénéplaine d'altitude moyenne 270-300 m et comprend à l'extrême Sud-Ouest le plus haut sommet du pays : le mont Ténakourou (747 m). Les sols rencontrés sont gravillonnaires, hydromorphes et ferralitiques. La végétation est dominée par des savanes boisées et des forêts claires, et parsemée de galeries forestières.

La population du Kénédougou était de 285695 habitants en 2006. Elle est jeune (plus de $50 \%$ a moins de 20 ans) avec une croissance démographique de 3,7 \% par an. Sa densité moyenne est de 24 habitants au $\mathrm{km}^{2}$. Dans chaque village de la province, il y a une communauté majoritaire, le plus souvent autochtone, et quelques communautés de migrants. Les communautés majoritaires sont les Sénoufo, les Toussian, les Bolon, et les Siamou dont les villages couvrent respectivement $55 \%, 16 \%, 12 \%$ et $6 \%$ du territoire provincial. Les groupes communautaires migrants dominants sont les Moose et les Peulh dont la proportion globale est de $1 \%$.

La principale activité économique est l'agriculture de rente et de subsistance qui occupe plus de $95 \%$ de la population de la province. L'élevage constitue la deuxième activité et se pratique en transhumance du Nord vers le Sud pendant la saison sèche et inversement dès l'installation des pluies. La cueillette des PFNL, qui est également très développée dans les villages, est surtout pratiquée par les femmes. Les produits les plus exploités sont les amandes de karité (Vitellaria paradoxa), les fruits du néré (Parkia biglobosa (Jacq.) R. Br. ex G. Don f.), les fruits et les feuilles du baobab (Adansonia digitata L.) et ceux du tamarinier (Tamarindus indica L.). 


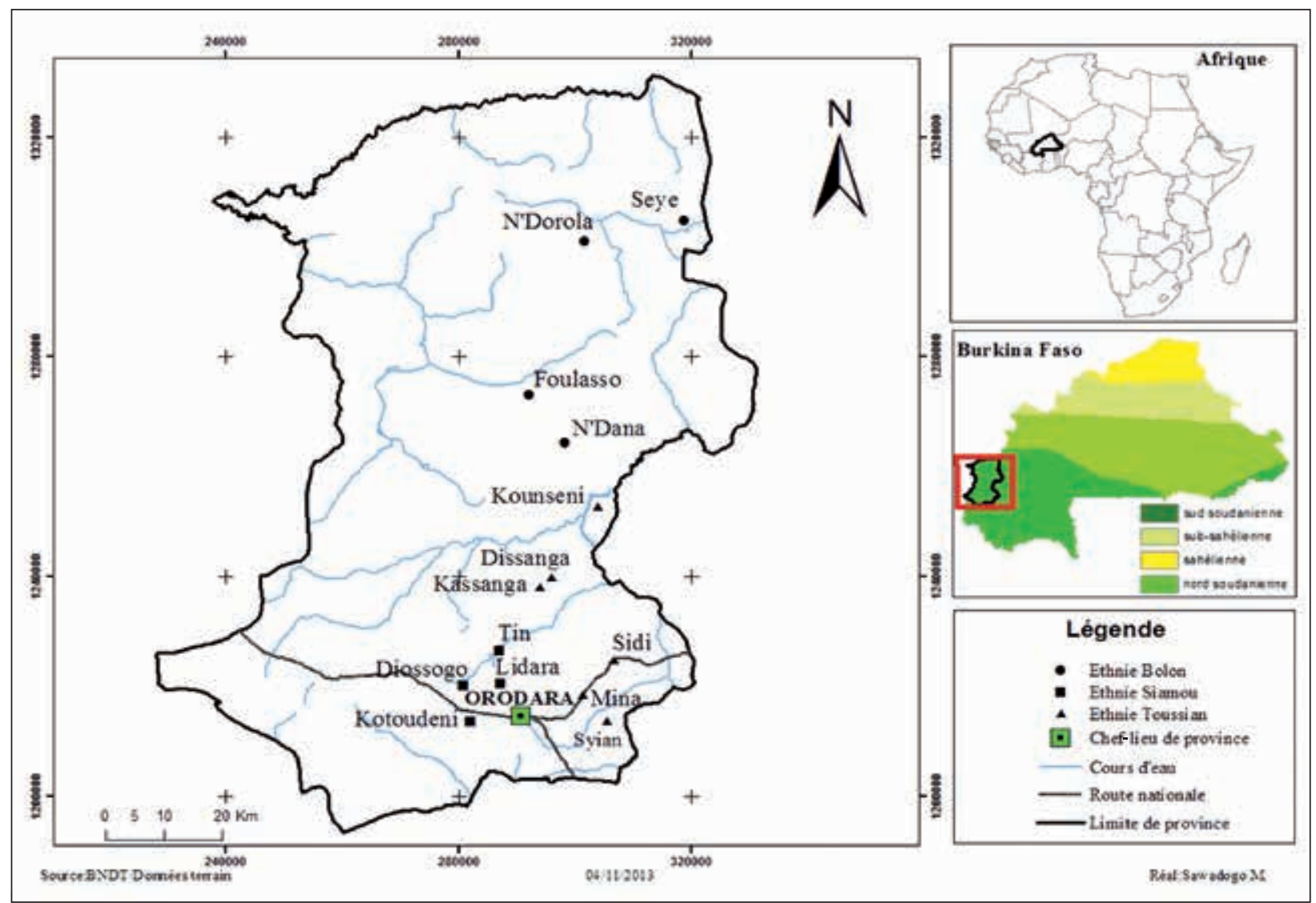

Figure 1.

Localisation de la zone d'étude.

\section{Échantillonnage et collecte de données}

Les investigations ont été menées dans 14 villages répartis sur l'ensemble de la province, soit un taux de sondage de $23 \%$. La méthode d'échantillonnage employée est de type aléatoire stratifié, basée sur trois groupes communautaires autochtones. Ce sont les Siamou, les Toussian et les Bolon. La communauté majoritaire Sénoufo n'a pas été prise en compte dans la présente étude car elle a fait l'objet d'une investigation similaire dans la province du Kénédougou (Ouédraogo et al., 2013). Pour les enquêtes ethnobotaniques, les villages ont été choisis de façon aléatoire à raison de quatre pour les communautés Siamou (Tin, Kotoudéni, Lidara et Diossogo), Bolon (N'Dorola, Seye, Foulasso et N'Dana) et six pour les Toussian (Kassanga, Dissanga, Syian, Mina, Sidi et Kounséni). Dans chaque village, 12 individus autochtones et 12 autres du groupe communautaire migrant Peulh ont été choisis et interviewés. Le genre et l'âge ont été pris en compte, à raison de trois générations par sexe : les jeunes âgés de moins de 20 ans, les adultes âgés de 20 à 50 ans et les aînés âgés de plus de 50 ans. Le nombre d'entretiens par tranche d'âge de chaque sexe a été de deux personnes. Au total, 336 questionnaires individuels ont été administrés à 168 femmes et 168 hommes à l'issue des interviews. Concernant l'approche participative sociale, vingt-huit entretiens de groupe ont été réalisés, avec les hommes et les femmes séparément pour éviter une influence du genre (Wenthol et al., 2001).

Des guides d'entretiens semi-directifs ont été utilisés lors des entretiens de groupe pour collecter les informations portant sur les espèces oléagineuses connues, les préférences des huiles, les critères influençant ces préférences et le poids de chaque critère. Au cours des entretiens individuels, les questions ont aussi concerné les domaines d'utilisation des huiles, les techniques d'extraction et de transformation des huiles, les huiles disponibles et utilisées localement et les actions menées pour la préservation des espèces oléagineuses. Les entretiens ont été réalisés avec l'aide de traducteurs et la reconnaissance des espèces citées a été possible grâce à la technique du guide voyageur (Albuquerque et al., 2006). Cette technique consiste :

- d'abord à noter les noms locaux des espèces connues et citées par la population et/ou l'individu questionné ;

- puis à accompagner un membre de la population ou l'individu concerné dans l'habitat naturel de la plante citée pour une observation directe ; à cette étape, un échantillon d'herbier est prélevé ;

- enfin à déterminer le nom scientifique de l'espèce par utilisation d'une flore. 
Les espèces répertoriées ont été obtenues par des citations spontanées des informateurs. Elles n'ont été considérées comme plantes locales que si elles sont spontanées au Burkina Faso et y poussent naturellement à l'état sauvage.

\section{Analyse et traitement des données}

Les données collectées ont servi à calculer les paramètres ci-après.

- La fréquence relative de citation des espèces selon les catégories de personnes interviewées : $F=(\mathrm{Nec} / \mathrm{N}) \times 100$. $\mathrm{Nec}$ représente le nombre de personnes ayant cité une espèce et $\mathrm{N}$ est le nombre total de personnes interviewées. Nous retenons le seuil de $20 \%$ pour distinguer les espèces les moins citées de celles les plus citées.

- L'Indice de possession de savoir global des espèces (IPSG) par communauté (formule adaptée de Assogbadjo et al., 2011) : IPSG $=\mathrm{Vm} / \mathrm{Nt}$. Vm est le nombre moyen des espèces citées par une communauté et $\mathrm{Nt}$ le nombre total des espèces citées par l'ensemble des communautés. Plus l'indice tend vers 1 et plus la majorité des personnes enquêtées de la communauté connaissent les espèces citées.

- L'Indice spécifique (Ins) des critères d'appréciation des espèces. Il est basé sur les critères qui déterminent le choix des huiles et renseigne par conséquent sur la préférence des espèces citées. Il varie de o à 1 et l'espèce qui a l'indice le plus élevé est la plus préférée. Pour le calcul de cet indice, une liste des critères déterminant le choix des huiles par les communautés autochtones a d'abord été dressée dans chaque village lors des entretiens groupés. Pour chaque critère cité, sa fréquence de citation a été calculée selon la formule suivante $: \mathrm{Fci}=\mathrm{Nci} / \mathrm{Nv}$, où $\mathrm{Nci}$ est le nombre de citations du critère i dans les entretiens groupés et Nv le nombre total de villages. Les critères retenus sont ceux qui ont une fréquence de citation au moins égale à $50 \%$. Ensuite, nous avons permis aux communautés d'attribuer une note (Belem et al., 2008) comprise entre 1 et 3 à chaque critère suivant son importance croissante. Pour chacun de ces critères, un indice Ini est calculé selon la formule $\Sigma$ Pnj / $\Sigma$ Pij. P est la note attribuée, Pnj représente la note du critère i donnée dans le village $j, n$ est le nombre de critères retenus, i varie de 1 à $n, j$ varie de 1 à 14 . Cet indice montre la participation de chaque critère à la préférence de l'espèce. Enfin, Ins est calculé pour chaque espèce suivant la formule Ins = $\Sigma \operatorname{lni}$ (1 ou o)) à partir d'une matrice espèces-critères de choix des huiles (tableau I).

- La fréquence d'utilisation relative des huiles (FURH) (formule adaptée de Camou-Guerrero et al., 2008) : FURH $=(\mathrm{Nuh} / \mathrm{Ntu}) \times 100$ où Nuh représente le nombre de citations d'utilisations de l'huile et Ntu le nombre de citations de tous les usages de l'espèce. Si FURH > $50 \%$, alors l'exploitation de l'espèce est orientée vers l'usage de l'huile.

- La valeur d'utilisation de l'espèce VU qui représente le niveau d'utilisation d'une espèce par les communautés (formule simplifiée de Cotton, 1996) : VU $=(\Sigma U i) / U t$ où Ui désigne le nombre d'utilisations différentes d'une espèce et Ut le nombre total de personnes ayant cité l'espèce.

- Le Facteur consensus d'informateurs ICF pour apprécier les accords entre les personnes interrogées sur les actions de préservation des espèces identifiées, menées dans les villages. Il certifie la présence véritable de l'action dans le village. La formule de Teklehaymanot (2009) a été adaptée à notre contexte : ICF = (Nap - Naa) / $(\mathrm{Nap}-1)$. Nap désigne les citations d'action de gestion menée dans le village et Naa les citations d'absence d'action dans le village. ICF varie entre o et 1. Une valeur proche de zéro indique un désaccord sur la connaissance des actions menées. Dans ce cas, les individus n'adhèrent pas à l'application des actions. Les actions de préservation adoptées par les personnes enquêtées concernent la restauration des peuplements par la plantation, l'interdiction de coupe de l'arbre en maintenant les arbres dans les vergers et l'entretien des arbres (pas de coupe de bois).

Les fréquences ont été comparées à travers le test non paramétrique de Wilcoxon / Kruskal-Wallis appliqué aux données anormalement distribuées. Les indices ont été également comparés au moyen du test paramétrique du " khi-deux ». Cela a permis d'apprécier les différences des valeurs moyennes des variables entre les catégories objets des enquêtes dans le logiciel R. Le logiciel Community Analysis Package (CAP) a servi pour une analyse factorielle de correspondance (AFC) afin d'apprécier les liens entre les groupes communautaires et les espèces oléagineuses citées en termes de savoirs.

Tableau I.

Matrice espèces-critères de choix des huiles.

\begin{tabular}{|c|c|c|c|c|c|c|}
\hline Espèces & $\mathrm{C}_{1}$ & $\mathrm{C}_{2}$ & C... & $\mathrm{Cn}$ & Ins & Rang \\
\hline $\mathrm{E}_{1}$ & In $1 \times(1$ ou 0$)$ & In $2 \times(1$ ou o) & $\ldots$ & In $n \times(1$ ou o) & $\sum$ Ini & \\
\hline E2 & $\ln 1 \times(1$ ou 0$)$ & In $2 \times(1$ ou 0$)$ & $\ldots$ & In $n \times(1$ ou o) & $\sum \operatorname{lni}$ & \\
\hline E3 & In $1 \times(1$ ou 0$)$ & In $2 \times(1$ ou 0$)$ & $\ldots$ & In $n \times(1$ ou o) & $\sum \operatorname{lni}$ & \\
\hline$\ldots$ & $\ldots$ & $\ldots$ & $\ldots$ & $\ldots$ & $\ldots$ & \\
\hline
\end{tabular}

$\mathrm{C}_{1}, \mathrm{n}$ : critère de sélection ; Ins : modèle de prévision des préférences des espèces ; Ini : indice calculé en tenant compte de la note attribuée au critère dans un village et du nombre de critères retenus. 1 = l'huile de l'espèce vérifie le critère ; 0 = l'huile de l'espèce ne vérifie pas le critère ; $C=$ critère ; $E=$ espèce. 


\section{Résultats \\ Savoirs locaux sur les plantes oléagineuses locales}

Au total, 11 espèces oléagineuses ont été citées par les 336 personnes enquêtées. Les quatre espèces majoritairement citées représentent $94 \%$ de l'ensemble des citations (1139). Ce sont Vitellaria paradoxa, Elaeis guineensis, Carapa procera DC. et Lophira lanceolata Tiegh. ex Keay (figure 2).

Parmi les groupes communautaires, les Siamou sont ceux qui possèdent le plus de savoirs sur les plantes oléagineuses (IPSG $=39,2 \%$ ), suivis des Toussian (33,5\%), des Bolon $(28,6 \%)$ et des Peulh $(27,9 \%)$. Toutefois, les différences ne sont pas significatives entre les groupes communautaires $\left(X^{2}=2,54 ; p=0,4678\right)$. Les résultats de l'AFC révèlent une cartographie des savoirs sur les plantes oléagineuses en fonction de la répartition géographique des groupes communautaires (figure 3). En effet, l'axe 1 du plan factoriel discrimine les Bolon des trois autres communautés. Les Bolon sont géographiquement éloignés des autres et citent des espèces que ces derniers ne mentionnent pas. Ce sont Sclerocarya birrea (A. Rich.) et Detarium microcarpum Guill. \& Perr., Borassus akeassii Bayton, Ouédr. \& Guinko. L'axe 2 discrimine les Peulh et les Toussian des Siamou qui connaissent et utilisent des espèces que les deux premières communautés ne citent pas. Il s'agit de Pentadesma byturacea Sabine et Lannea microcarpa Engl. \& K.Krause. De façon générale, en plus des espèces communes aux quatre communautés, les Siamou et les Bolon connaissent des espèces spécifiques à leurs terroirs.

Indépendamment des groupes communautaires, le savoir sur les espèces rarement citées varie significativement suivant l'âge $\left(X^{2}=8,11 ; p=0,0173\right)$. En effet, le test de Wilcoxon / Kruskal-Wallis révèle une différence significative des fréquences de citation de ces espèces entre les jeunes et les adultes $(Z=-2,53 ; p=0,0115)$, d'une part, et entre les jeunes et les aînés $(Z=2,37 ; p=0,0175)$ d'autre part, alors que celles des adultes et des aînés sont comparables

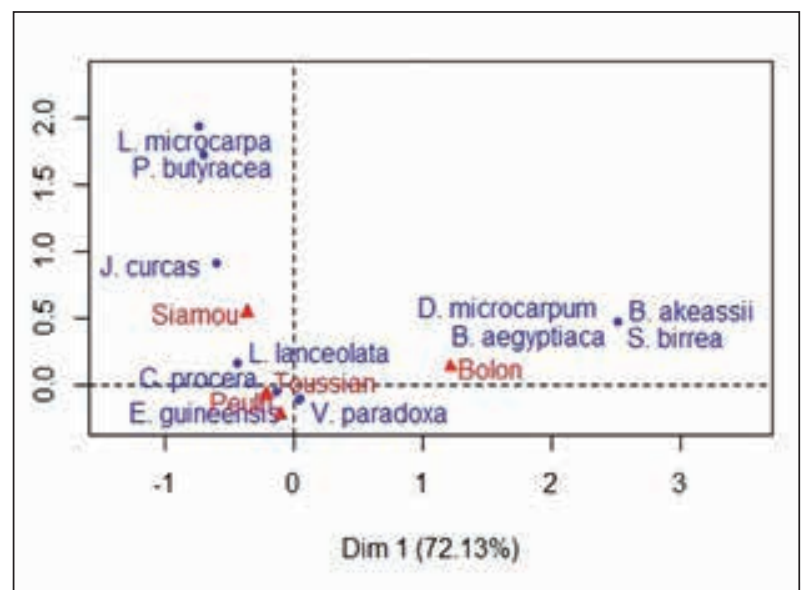

Figure 3.

AFC affectant les espèces citées aux groupes communautaires.

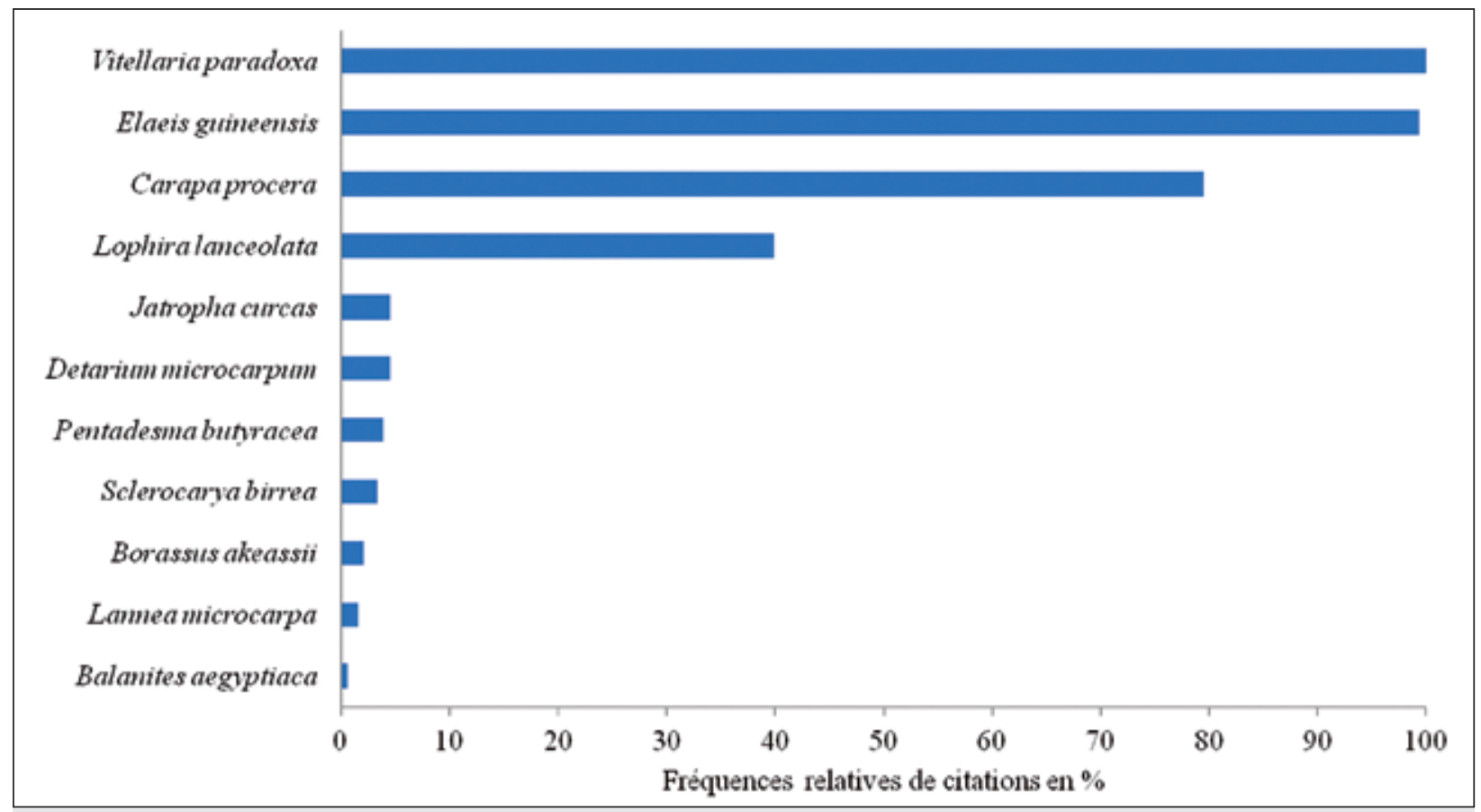

Figure 2.

Classification des espèces selon la fréquence relative de leur citation. 
$(Z=0,79 ; p=0,4275)$. Parmi les sept espèces les moins citées, seulement trois sont citées par les jeunes alors que les adultes et les aînés en connaissent six. Les savoirs ne varient pas significativement suivant le genre au sein des générations $\left(X^{2}=0,05 ; p=0,8167\right)$. Une seule espèce est connue uniquement par les jeunes : Balanites aegyptiaca.

\section{Savoirs sur l'extraction et la transformation des huiles}

Les savoirs sur les techniques d'extraction de l'huile brute et sa transformation en produits cosmétiques varient suivant les espèces. Les populations savent extraire l'huile de la plupart des graines des espèces citées à l'exception de Borassus akeassii Bayton, Ouédr. \& Guinko, Lannea microcarpa et Balanites aegyptiaca (L.) Del. Les savoirs dépendent du genre et des générations. Les techniques d'extraction des huiles sont détenues par $23 \%$ des personnes interviewées dont $95 \%$ sont constituées de femmes $\left(X^{2}=19,79 ; p<0,001\right)$. Parmi les femmes, le savoir sur l'extraction des huiles varie entre les générations (42 \% chez les aînées, 33 \% chez les adultes et $25 \%$ chez les jeunes) (photo 2 ).

Pour ce qui concerne la transformation de l'huile, $22 \%$ des personnes interviewées disposent de savoirs sur les procédés de fabrication de produits cosmétiques (savon et huile pour les soins de la peau). Parmi celles-ci, les femmes sont significativement majoritaires avec une proportion de $71 \%$ $\left(X^{2}=13,58 ; p<0,001\right)$. Les procédés d'utilisation médicinale des huiles sont connus par $75 \%$ des personnes enquêtées sans distinction de genre $\left(X^{2}=0,08 ; p=0,7727\right)$. Toutefois, ces savoirs régressent des aînés vers les jeunes avec des fréquences de citation respectives de $43 \%$ et $17 \%$.

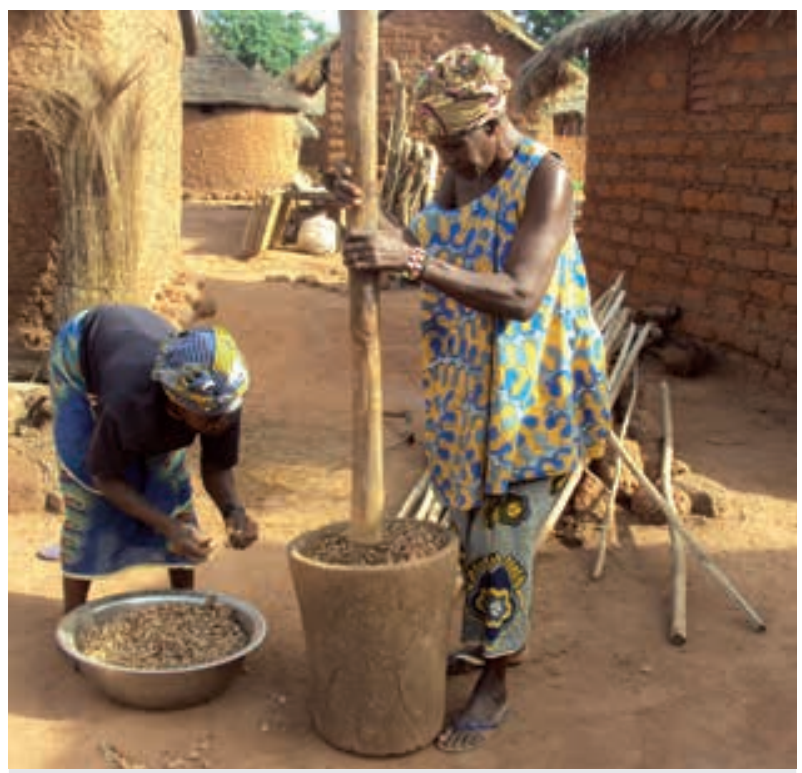

Photo 2.

Pilage des fruits pour l'extraction d'huile de Lophira lanceolata par les femmes.

Photo F. R. Tiétiambou.

\section{Utilisations des huiles}

Parmi les 11 espèces oléagineuses identifiées par les communautés rurales, sept ont les techniques d'extraction de leur huile bien connues. Ce sont Vitellaria paradoxa, Elaeis guineensis, Carapa procera, Sclerocarya birrea, Pentadesma butyracea, Lophira lanceolata et Detarium microparpum. Toutefois, seules les huiles de Vitellaria paradoxa, Elaeis guineensis, Carapa procera et Lophira lanceolata sont effectivement utilisées par les groupes communautaires dans l'autoconsommation (photos 3 et 4 ). Pour les 11 espèces, les huiles sont utilisées dans la médecine (47\%), l'alimentation (37\%) et la cosmétique (16\%). Parmi les 10 espèces citées, Balanites aegyptiaca est certes reconnue comme une plante oléagineuse mais les utilisations de son huile sont quasiment méconnues. Les utilisations des huiles des neuf autres espèces se fait dans l'ordre de préférence suivante : l'alimentation (56\%), la médecine (34\%) et la cosmétique (10\%) (figure 4).

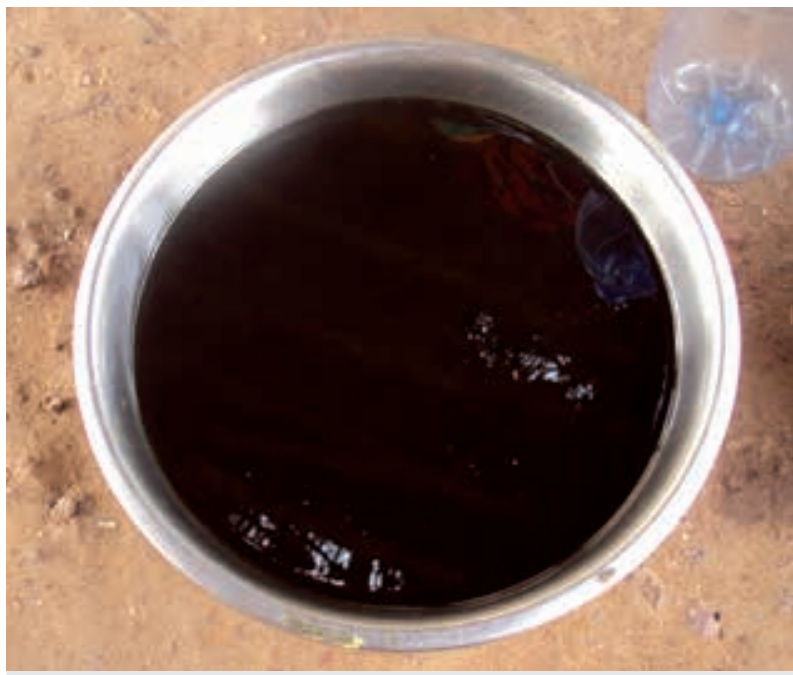

Photo 3.

Huile extraite des graines de Lophira lanceolata.

Photo F. R. Tiétiambou.

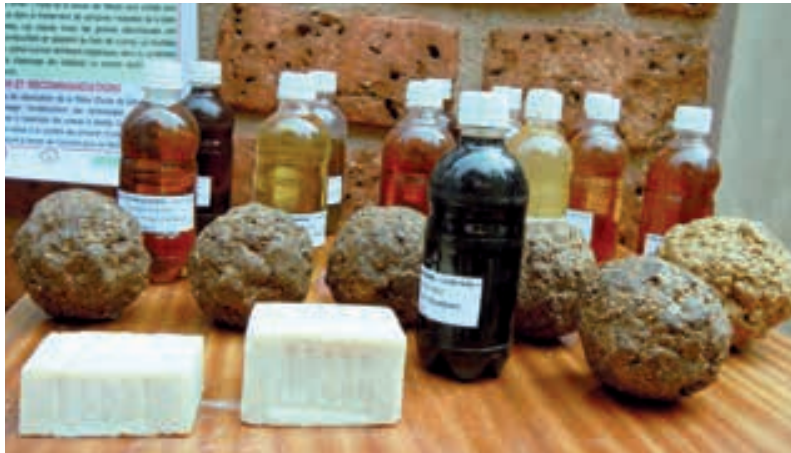

Photo 4 .

Huiles et savons traditionnellement produits à base des graines oléagineuses de Carapa procera.

Photo A. Ouédraogo. 


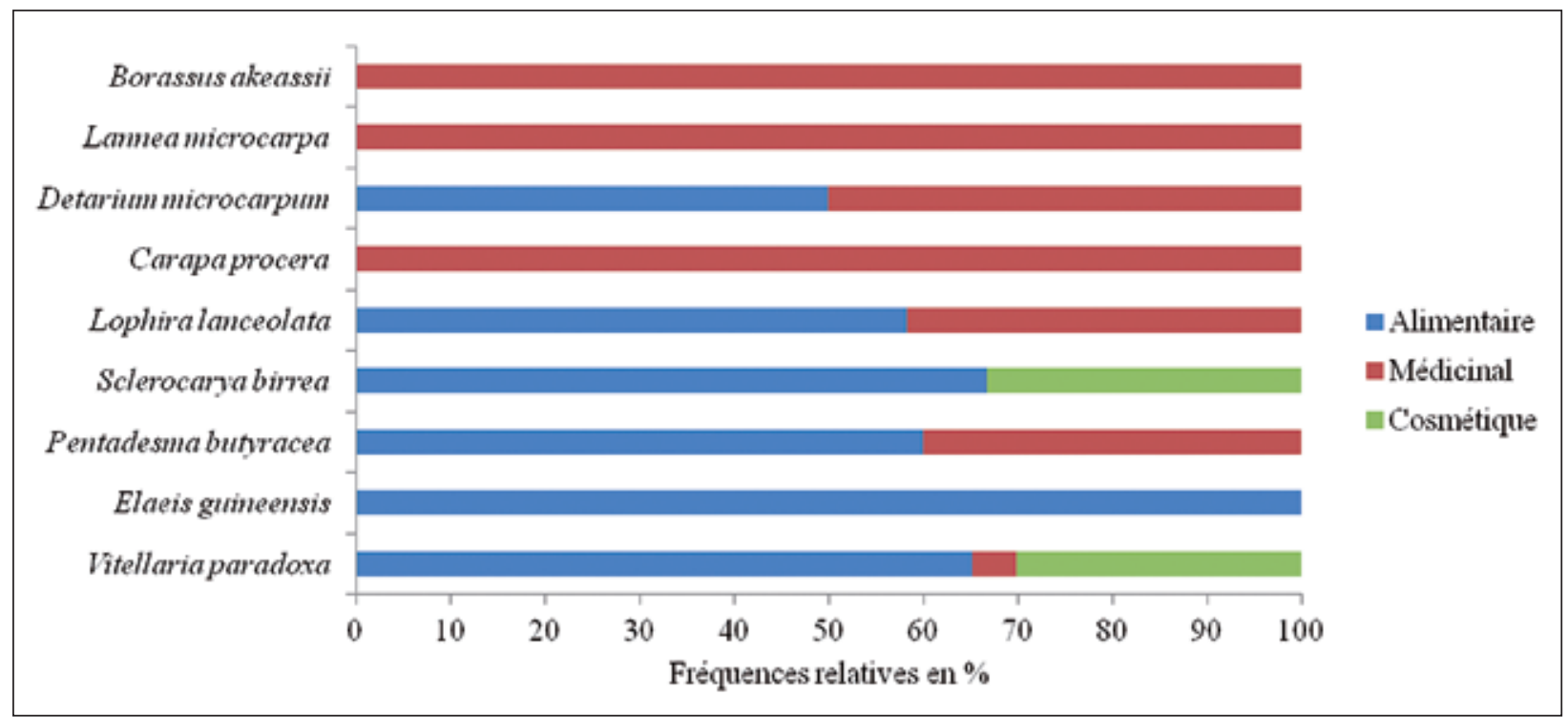

Figure 4.

Importance des préférences d'utilisation des huiles d'arbres locaux pour les préparations médicinales, alimentaires et cosmétiques.

L'huile de Vitellaria paradoxa peut être utilisée dans tous ces domaines. Cependant, certaines huiles sont citées uniquement pour une seule utilisation de préférence. C'est le cas de Borassus akeassii, Lannea microcarpa, Carapa procera dans le domaine médicinal et Elaeis guineensis dans l'alimentation. Les espèces locales connues pour l'exploitation commerciale de leur huile sont Carapa procera (50\%), Sclerocarya birrea (40\%), Elaeis guineensis (33\%) et Vitellaria paradoxa (27\%).

La comparaison des valeurs moyennes de citations de l'utilisation des huiles entre les groupes communautaires révèle qu'il y a des différences significatives entre ces groupes $\left(X^{2}=17,90 ; p=0,0005\right)$. Les utilisations chez les Siamou et des Bolon à des fins commerciales sont comparables $(Z=-1,12 ; p=0,26)$ tout comme chez les Toussian et les Peulh $(Z=-0,44 ; p=0,65)$. Les Peulh citent plus les utilisations cosmétiques, alimentaires et médicinales des huiles (tableau II).

\section{Préférences et valeurs d'usage des plantes oléagineuses locales}

Sept critères sur 14 initialement énumérés ont été retenus sur la base de leurs fréquences comme étant les plus déterminants pour le choix des huiles par les communautés locales. Il s'agit de l'utilisation alimentaire (Al) (100\% des fréquences de citation), l'utilisation médicinale (Me) (100\%), le savoir sur la technique d'extraction de l'huile (Tc) (93\%), l'utilisation coutumière (Cu) (79\%), l'utilisation cosmétique (CS) (71\%), le rendement en huile extractible de la graine (R) (71\%) et la facilité de la technique d'extraction (Tf) (64\%). Les indices de chaque critère sont donnés dans l'équation modèle de prévision des préférences des espèces suivante : Ins $=0,15(\mathrm{Cu})+0,25(\mathrm{Al})+0,20(\mathrm{Me})+0,08(\mathrm{Cs})+0,14(\mathrm{Tc})+$ $0,08(T f)+0,10(R)$.
Tableau II.

Fréquences relatives de citation d'utilisation de l'huile par communauté.

\begin{tabular}{|l|c|c|c|c|}
\hline \multirow{2}{*}{} & \multirow{2}{*}{$\begin{array}{c}\text { Commercialisation } \\
\text { (\%) }\end{array}$} & \multicolumn{3}{|c|}{ Autoconsommation (\%) } \\
\cline { 3 - 5 } & & Alimentaire & Médicinale & Cosmétique \\
\hline Siamou & 7,2 & 15,8 & 14,3 & 5,7 \\
\hline Toussian & 1,3 & 23,8 & 14,0 & 7,5 \\
\hline Bolon & 7,0 & 15,0 & 8,7 & 7,0 \\
\hline Peulh & 3,7 & 56,0 & 34,5 & 29,8 \\
\hline
\end{tabular}

Le tableau III renseigne sur la valeur que peut prendre chaque critère influençant les choix chez l'ensemble des espèces. L'huile de l'espèce Vitellaria paradoxa vérifie tous les critères de choix suivie de Elaeis guinneensis.

Ainsi, le classement des espèces oléagineuses sur la base de ces critères est donné dans le tableau IV. À part Pentadesma butyracea qui est une espèce rare, les espèces oléagineuses les plus préférées (Ins > 0,5) correspondent à celles dont l'huile est localement disponible. Les valeurs d'utilisation indiquent que l'huile est peu exploitée pour l'ensemble des espèces, les meilleurs scores concernant les espèces bien connues. Elles montrent également que toutes les espèces oléagineuses ne sont pas exploitées uniquement pour leur huile. Les parties non oléagineuses sont aussi exploitées. Le tableau $\mathrm{V}$ indique que les huiles représentent $71 \%$ de l'ensemble des utilisations des espèces ; $76 \%$ du revenu de l'exploitation commerciale des espèces provient de l'huile. Parmi les productions autoconsommées des espèces, les huiles contribuent à $99 \%$ dans le domaine cosmétique. Dans le domaine médicinal, elles ne contribuent que de moitié (51\%). 
Tableau III.

Vérification des critères de choix des huiles des espèces oléagineuses.

\begin{tabular}{|c|c|c|c|c|c|c|c|}
\hline Espèces & Al & Me & Tc & $\mathrm{Cu}$ & Cs & $\mathbf{R}$ & Tf \\
\hline Carapa procera & 0 & 1 & 0 & 1 & 1 & 1 & 0 \\
\hline Detarium microparpum & 0 & 1 & 0 & 0 & 1 & 1 & 0 \\
\hline Elaeis guineensis & 1 & 1 & 1 & 0 & 1 & 1 & 1 \\
\hline Jatropha curcas & 0 & 1 & 0 & 0 & 1 & 0 & 0 \\
\hline Lannea microcarpa & 1 & 1 & 1 & 0 & 1 & 0 & 1 \\
\hline Lophira lanceolata & 1 & 1 & 0 & 0 & 1 & 0 & 1 \\
\hline Pentadesma butyracea & 1 & 1 & 0 & 0 & 1 & 1 & 1 \\
\hline Sclerocaya birrea & 0 & 1 & 0 & 0 & 1 & 1 & 0 \\
\hline Vitellaria paradoxa & 1 & 1 & 1 & 1 & 1 & 1 & 1 \\
\hline Borassus akeassii & 0 & 1 & 0 & 0 & 1 & 0 & 0 \\
\hline
\end{tabular}

$\mathrm{Al}=$ utilisation alimentaire ; Me = utilisation médicinale ; Tc = connaissance de la technique d'extraction de l'huile ;

$\mathrm{Cu}=$ utilisation coutumière ; $\mathrm{CS}=$ utilisation cosmétique ; $\mathrm{R}=$ rendement en huile extractible de la graine ;

Tf = facilité de la technique d'extraction ; 1 = l'huile de l'espèce vérifie le critère ; 0 = l'huile de l'espèce ne vérifie pas le critère.

\section{Tableau IV.}

Indices spécifiques et valeurs d'utilisation des espèces oléagineuses.

\begin{tabular}{|l|c|c|c|c|}
\hline Espèces & Fréquence de citation (\%) & Indice spécifique de préférence & VU huile & VU espèce \\
\hline Vitellaria paradoxa & 100 & 1,00 & 3,01 & 3,03 \\
\hline Elaeis guineensis & 99,4 & 0,85 & 1,15 & 2,15 \\
\hline Pentadesma butyracea & 3,9 & 0,70 & 0,00 & 1,0 \\
\hline Lophira lanceolata & 39,9 & 0,60 & 0,08 & 1,02 \\
\hline Carapa procera & 79,5 & 0,51 & 0,38 & 0,39 \\
\hline Sclerocarya birrea & 3,3 & 0,37 & 0,00 & 1,18 \\
\hline Detarium microcarpum & 4,5 & 0,37 & 0,00 & 0,87 \\
\hline Lannea microcarpa & 1,5 & 0,36 & 0,00 & 1,60 \\
\hline Borassus akeassii & 2,1 & 0,27 & 0,00 & 1,14 \\
\hline Jatropha curcas & 4,5 & 0,20 & 0,00 & 0,60 \\
\hline
\end{tabular}

VU $=$ valeur d'utilisation.

Tableau V.

Fréquences relatives d'utilisation des huiles par communauté.

\begin{tabular}{|l|l|c|c|c|c|c|}
\hline & & Siamou & Toussian & Bolon & Peulh & Globale \\
\hline Commercialisation (\%) & & 77 & 44 & 94 & 69 & 76 \\
\hline \multirow{3}{*}{ Autoconsommation (\%) } & Alimentaire & 96 & 95 & 79 & 94 & 92 \\
& Médicinale & 52 & 47 & 49 & 52 & 51 \\
& Cosmétique & 94 & 100 & 95 & 99 & 99 \\
\hline
\end{tabular}


Dans les villages, des actions d'entretien et d'interdiction de coupe des arbres concernent ces espèces et sont bien connues de la population (ICF > 50 \% pour tous les groupes communautaires). Quant à la restauration des peuplements, l'accord global est faible pour toutes les communautés $(I C F=0,32)$, traduisant un faible intérêt pour la plantation des espèces (tableau VI). Chez les Toussian, il y a un désaccord manifeste sur le reboisement des espèces $(I C F=0,04)$. Le niveau de participation aux actions de préservation varie en fonction de l'espèce. Chez tous les groupes communautaires, les espèces les plus utiles sont celles qui recueillent le plus d'adhésion pour leur préservation.

\section{Discussion}

\section{Savoirs sur les plantes oléagineuses et sur l'exploitation des huiles}

Même si les savoirs varient en fonction des groupes communautaires, ils sont aussi fortement liés à la disponibilité des espèces dans les terroirs. En effet, Traoré et al. (2011) rapportent que plus une espèce est présente et pousse naturellement dans une région, plus elle est accessible, connue et utilisée. Les espèces citées sont non seulement celles qui sont disponibles dans les villages mais également reconnues comme étant oléagineuses. Des espèces sont abondantes dans certains villages et rares ou absentes dans d'autres du fait de leurs exigences écologiques ; c'est le cas de Carapa procera et de Pentadesma butyracea qui sont des espèces de galeries forestières. Balanites aegyptiaca, elle, est rarement citée à cause de sa distribution plutôt sahélosoudanienne (Ouédraogo, 2006 ; Sagna et al., 2014) qui fait qu'elle est rare sous le climat sud-soudanien de la province du Kénédougou. Elle n'a été citée que par une faible proportion de jeunes (2\%). Ces jeunes l'ont connue car ils ont effectué un déplacement dans la zone d'extraction d'huile de Balanites aegyptiaca. En général, les aînés connaissent beaucoup plus d'espèces car ils ont acquis des savoirs auprès de leurs parents qui utilisaient les huiles dans divers domaines. De nos jours, les utilisations ancestrales tendent à disparaître avec la rareté des ressources (Weber et al., 2010).

Concernant les pratiques de l'extraction de l'huile, ce sont plus les femmes qui en détiennent le savoir-faire. Le contrôle féminin du savoir est également rapporté à propos de la production du beurre de karité (Saussey et al., 2008). Selon Elias et Carney (2007), les femmes sont les principales détentrices des savoirs d'utilisations domestiques. La transmission intergénérationnelle du savoir rencontre des contraintes liées au fait que les jeunes s'intéressent de moins en moins aux pratiques traditionnelles jugées désuètes et moins rentables. Weber et al. (2010) ont fait le même constat pour ce qui concerne l'extraction de l'huile de Carapa procera au Mali et au Sénégal. Toutefois, la transmission du savoir varie en fonction des espèces de plantes. Cela est confirmé par Ouédraogo et al. (2013) qui rapportent que la non-exploitation de certaines espèces oléagineuses s'explique par la méconnaissance de la technique d'extraction de leur huile.
Tableau VI.

Facteurs du consensus d'informateurs par communauté.

\begin{tabular}{|l|c|c|c|}
\hline & Entretien & Restauration & Interdiction \\
\hline Siamou & 0,72 & 0,38 & 0,72 \\
\hline Toussian & 0,59 & 0,04 & 0,57 \\
\hline Bolon & 0,86 & 0,71 & 0,87 \\
\hline Peulh & 0,66 & 0,33 & 0,68 \\
\hline Global & 0,68 & 0,32 & 0,68 \\
\hline
\end{tabular}

Le mode oral de transmission des savoirs rend l'information vulnérable sur le long terme (Saussey et al., 2008) et remet également en cause sa fiabilité (De Caluwé et al., 2009). Les savoirs conservés se limitent souvent à ceux qui répondent mieux à l'environnement des générations jeunes. C'est dans cette perspective qu'Olivier de Sardan (1995) soutient la contextualisation des savoir-faire locaux empiriques. Dans le cas de la production du beurre de karité dans l'espace domestique, les femmes suivent un système de règles lignagères et générationnelles qui privilégient certaines formes de coopération ; les plus jeunes constituant la source de travail, les femmes en âge de procréer la source de capital et les femmes ménopausées la source de connaissance (Chalfin, 2004). Cette implication de différentes générations dans les étapes de production est une garantie pour la conservation des savoirs locaux.

\section{Préférences et valeurs d'usage des plantes oléagineuses locales}

Les communautés locales utilisent les huiles dans trois domaines principaux : alimentation, cosmétique et médecine. Parmi les critères qui déterminent les préférences des huiles par les communautés locales, l'utilisation alimentaire et médicinale reste commune à toutes les espèces. En général, ces critères sont indépendants du nombre d'utilisations des espèces mais ils conditionnent l'exploitation de ces dernières. C'est le cas de Pentadesma butyracea qui est une espèce rare et relativement peu connue au Burkina Faso (Lebrun et Stork, 1991) mais qui figure parmi les espèces préférées. Les préférences sont orientées vers les huiles qui sont bien connues et couramment utilisées (Ouédraogo et al., 2013). Le désintérêt pour certaines plantes oléagineuses est dû à la faible teneur en huile de leurs graines (cas de Detarium microcarpum) et de la difficulté d'extraction (Kyari, 2008). Pour la population locale, la valeur marchande des espèces oléagineuses dépend de leur niveau de préférence. Par conséquent, la génération de revenus par une espèce est une conséquence et non un critère de préférence des populations. Ce constat corrobore celui de Garibaldi et Turner (2004) qui ont montré que l'importance d'une espèce ne se limite pas à son exploitation commerciale. Toutefois, Lamien et Traoré (2002) soutiennent que la promotion de la biodiversité et la régénération des espèces passent par leur domestication à travers le développement du marché de leurs produits non ligneux. Mis à part le karité (Vitellaria paradoxa) et le palmier à huile (Elaeis guineensis) qui sont bien connus et exploités par la 
population, les huiles de Lophira lanceolata, Carapa procera et Pentadesma butyracea sont fréquemment citées dans de nombreuses utilisations. Ouédraogo et al. (2013) soutiennent que ces trois espèces oléagineuses ont un potentiel de valorisation. Carapa procera n'a été citée que dans quatre des 14 villages enquêtés (Kotoudeni, Tin, Diessogo et Syian), lesquels sont traversés par un cours d'eau permanent sur les rives duquel poussent quelques peuplements de cette plante. L'huile de cette espèce n'est pas alimentaire mais elle est utilisée en médecine et cosmétique. Cela a été également rapporté par Weber et al. (2010) qui mentionnent l'utilisation traditionnelle de l'huile de Carapa procera au Mali et au Sénégal pour traiter diverses maladies, produire du savon et soigner la peau. Nos résultats montrent que l'huile de Lophira lanceolata est utilisée dans l'alimentation et en médecine traditionnelle. Fariku et Kidah (2008) et Lohlum et al. (2010) rapportent que les graines de l'espèce contiennent jusqu'à $50 \%$ d'huile à usage alimentaire et cosmétique. L'huile de Pentadesma butyracea est citée dans les utilisations alimentaires et médicinales tandis qu'au Bénin, Avocèvou-Ayisso et al. (2012) rapportent qu'elle est en plus employée en cosmétique. L'importance de l'utilisation cosmétique de l'huile de Pentadesma butyracea est aujourd'hui reconnue partout dans le monde et peut constituer une bonne opportunité pour la promotion de l'espèce (Gruenwald et Galizia, 2005).

\section{Conclusion}

Les résultats de la présente étude indiquent que les communautés Siamou, Bolon, Toussian et Peulh du Kénédougou connaissent une dizaine de plantes oléagineuses locales avec des utilisations diversifiées de leur huile. Toutefois, les savoirs endogènes sont variables selon les catégories sociolinguistiques. La promotion des espèces sous-exploitées à des fins commerciales (Carapa procera) ou alimentaires (Lophira lanceolata et Pentadesma butyracea) peut contribuer à améliorer les conditions de vie des communautés. En termes de gestion durable des ressources, il est évident que la population sera plus disposée à conserver et exploiter rationnellement ces plantes locales si la valeur d'utilisation de leurs produits est avérée. Par conséquent, des stratégies de gestion axées sur la valorisation doivent être appliquées à toutes ces espèces afin de garantir leur exploitation durable. Le potentiel socio-économique de ces espèces locales pourrait alors se développer en s'appuyant sur un marché de produits oléagineux qui va absorber la production locale d'huile et de produits oléagineux. Les espèces oléagineuses locales peuvent constituer ainsi une source d'emplois et de diversification des revenus. Une demande importante en huile et en produits oléagineux pourrait être une opportunité pour promouvoir la domestication de ces espèces locales.

\section{Remerciements}

Les auteurs remercient Danida qui a financé ces travaux à travers le projet QualiTree (DFC, projet $\left.n^{\circ} 10-002 \mathrm{AU}\right)$.
Ils sont également reconnaissants à la population locale des quatorze villages.

\section{Références bibliographiques}

APFNL, 2011. Stratégie nationale de valorisation et de promotion des produits forestiers non ligneux. Ouagadougou, Burkina Faso, MEDD, Agence des produits forestiers non ligneux, $74 \mathrm{p}$.

Albuquerque U. P., Lucena R. F., Monteiro J. M., Florentino A. T., Almeida C. B., 2006. Evaluating two quantitative ethnobotanical techniques. Ethnobotany Research and Applications, 4: 51-60.

Assogbadjo A., Glèlè-Kakaï R., Adjallala F., Azihou A., Vodouhê G., Kyndt T., Codjia J., 2011. Ethnic differences in use value and use patterns of the threatened multipurpose scrambling shrub (Caesalpinia bonduc L.) in Benin. Journal of Medicinal Plants Research, 5 (9): 1549-1557.

Avocèvou-Ayisso C., Avohou T. H., Oumorou M., Dossou G., Sinsin B., 2012. Ethnobotany of Pentadesma butyracea in Benin: A quantitative approach. Ethnobotany Research and Applications, 9: 151-166.

Belem B., Smith Olsen C., Theilade I., Bellefontaine R., Guinko S., Lykke A. M., Diallo A., Boussim J. I., 2008. Identification des arbres hors forêt préférés des populations du Sanmatenga (Burkina Faso). Bois et Forêts des Tropiques, 298 (4) : 53-60. http://bft.cirad.fr/cd/BFT_298_53-60.pdf

Bognounou F., Thiombiano A., Savadogo P., Boussim J. I., Odén P. C., Guinko S., 2009. Wood vegetation structure and composition at four sites along a latitudinal gradient in Western Burkina Faso. Bois et Forêts des Tropiques, 300 (2): 2944. http://bft.cirad.fr/cd/BFT_300_29-44.pdf

Camou-Guerrero A., Reyes-García V., Martínez-Ramos M., Casas A., 2008. Knowledge and Use Value of Plant Species in a Rarámuri Community: A Gender Perspective for Conservation. Human Ecology, 36: 259-272.

Chalfin B., 2004. Shea butter republic: State power, global markets, and the making of an indigenous commodity. Londres, Royaume-Uni, Routledge, 300 p.

Cotton C. M., 1996. Ethnobotany: Principles and Applications. Chichester, Royaume-Uni, John Wiley \& Sons, 424 p.

Datta S. K., Pandey R. K., 1993. Applied Botany Abstracts, 1 (2): 108-118.

De Caluwé E., De Smedt S., Assogbadjo A. E., Samson R., Sinsin B., Van Damme P., 2009. Ethnic differences in use value and use patterns of baobab (Adansonia digitata L.) in northern Benin. African Journal of Ecology, 47: 433-440.

Elias M., Carney J., 2007. African shea butter: A feminized subsidy from nature. Africa, 77: 37-62.

Fariku S., Kidah M., 2008. Biomass potential of Lophira lanceolata fruit as a renewable energy resource. African Journal of Biotechnology, 7 (3): 308-310. 
Fontès J., Guinko S., 1995. Carte de la végétation et de l'occupation du sol du Burkina Faso. Notice explicative. Toulouse, France, Ministère de la Coopération, Projet Campus, 68 p.

Garibaldi A., Turner N., 2004. Cultural keystone species: Implications for conservation and restoration. Ecology and Society, 9 (3): art. 1.

Gruenwald J., Galizia M., 2005. Market brief in the European Union for selected natural ingredients derived from native species. Adansonia digitata L., Baobab. United Nations Conference on Trade and Development, Genève, Suisse.

Kaboré S. A., Bastide B., Traoré S., Boussim J. I., 2012. Dynamique du karité, Vitellaria paradoxa, dans les systèmes agraires du Burkina Faso. Bois et Forêts des Tropiques, 313 (3) : 47-59. http://bft.cirad.fr/cd/BFT_313_47-59.pdf

Kyari M. Z., 2008. Extraction and characterization of seed oils. International Agrophysics, 22: 139-142.

Lamien N., Traoré S., 2002. Commercialisation des produits forestiers non ligneux dans la zone semi-aride du Burkina Faso : cas des feuilles sèches de baobab (Adansonia digitata L.). Bamako, Mali, Icraf, 12 p.

Lebrun J.-P., Stork A. L., 1991. Énumération des plantes à fleurs d'Afrique tropicale. Volume I : Généralités et Annonaceae à Euphorbiaceae et Pandaceae. Genève, Suisse, Conservatoire et Jardin botaniques de la ville de Genève.

Lohlum S. A., Maikidi G. H., Salomon M., 2010. Proximate composition, amino acid profile and phytochemical screening of Lophira lanceolata seeds. African Journal of Food, Agriculture, Nutrition and Development, 10 (1): 1-12.

Lynam T., Cunliffe R., Mapaure I., 2004. Assessing the importance of woodland landscape locations for both local communities and conservation in Gorongosa and Muanza Districts, Sofala Province, Mozambique. Ecology and Society, 9 (4): art. 1.

Mathur M., Sundaramoorthy S., 2013. Census of Approaches Used in Quantitative Ethnobotany. Ethno Medicine, 7 (1): 31-58.

Mbayngone E., Schmidt M., Hahn-Hadjali K., Thiombiano A., Guinko S., 2008. Magnoliophyta of the partial faunal reserve of Pama, Burkina Faso. Check List, 4 (3): 251-266.

MEF (Ministère de l'Économie et des Finances), 2010. Stratégie de croissance accélérée et de développement durable. Ouagadougou, Burkina Faso, Ministère de l'Économie et des Finances, $112 \mathrm{p}$.

Ouédraogo A., 2006. Diversité et dynamique de la végétation ligneuse de la partie orientale du Burkina Faso. Thèse de doctorat, Université de Ouagadougou, Burkina Faso, 195 p.

Ouédraogo A., Lykke A. M., Lankoandé B., Korbéogo G., 2013. Potentials for Promoting Oil Products Identified from Traditional Knowledge of Native Trees in Burkina Faso. Ethnobotany Research Applications, 11: 71-83.

Ouédraogo I., Nacoulma B. M. I., Ouédraogo O., Hahn K., Thiombiano A., 2014. Productivité et valeur économique des calices de Bombax costatum Pellegr. \& Vuillet en zone soudanienne du Burkina Faso. Bois et Forêts des Tropiques, 319 (1) : 31-41. http://bft.cirad.fr/cd/BFT_319_31-41.pdf

Olivier de Sardan J.-P., 1995. Anthropologie et développement. Essai en socio-anthropologie du changement social. Marseille, Paris, France, Apad/Karthala, 221 p.

Sagna M., Niang K., Goffner D., 2014. Balanites aegyptiaca (L.) Delile : distribution géographique et connaissances ethnobotaniques des populations locales du Ferlo (Nord Sénégal). Biotechnologie, Agronomie, Société et Environnement, $18:$ 503-511.

Saussey M., Moity-Maizi P., Muchnik J., 2008. Nouvelles formes de reconnaissance des femmes burkinabè dans la production collective de beurre de karité. Cahiers Agricultures, 17: 582-586.

Schmidt M., Kreft H., Thiombiano A., Zizka G., 2005. Herbarium collections and field data-based plant diversity maps for Burkina Faso. Diversity and Distributions, 11 (6): 509-516.

Schreckenberg K., 2004. The contribution of shea butter (Vitellaria paradoxa C.F. Gaertner) to local livelihoods in Benin. In: Forest Products, Livelihoods and Conservation. Case studies of non-timber forest product systems. Volume 2. Jakarta, Indonésie, Center for International Forestry Research, 91-114.

Sheil D., Liswanti N., 2006. Scoring the importance of tropical forest landscapes with local people: patterns and insights. Environmental Management, 38 (1): 126-136.

Teklehaymanot T., 2009. Ethnobotanical study of knowledge and medicinal plants use by the people in Dek Island in Ethiopia. Journal of Ethnopharmacology, 124 (1): 69-78.

Traoré L., Ouédraogo I., Ouédraogo A., Thiombiano A., 2011. Perceptions, usages et vulnérabilité des ressources végétales ligneuses dans le Sud-Ouest du Burkina Faso. International Journal of Biological and Chemical Sciences, 5 (1): 258-278.

Vodouhê F. G., Coulibaly O., Greene C., Sinsin B., 2009. Estimating the Local Value of Non-Timber Forest Products to Pendjari Biosphere Reserve Dwellers in Benin. Economic Botany, 63: 397-412.

Weber N., Birnbaum P., Forget P.-M., Gueye M., Kenfack D., 2010. L'huile de carapa (Carapa spp., Meliaceae) en Afrique de l'Ouest : utilisations et implications dans la conservation des peuplements naturels. Fruits, $65: 343-354$. http:// dx.doi.org/10.1051/fruits/2010029

Wenthol W., Dembélé A. R. K., Diallo M., 2001. Genre et recherche agricole au Mali. Amsterdam, Pays-Bas, KIT Publishers, $141 \mathrm{p}$.

Zerbo P., Millogo-Rasolodimby J., Nacoulma-Ouedraogo O. G., Van Damme P., 2011. Plantes médicinales et pratiques médicales au Burkina Faso : cas des Sanan. Bois et Forêts des Tropiques, 307 (1) : 41-53. http://bft.cirad.fr/cd/ BFT_307_41-53.pdf 